\title{
Changes In Long Term Neural Connectivity Following Psychological Trauma.
}

\author{
Fallon Cook ${ }^{1}$, Joseph Ciorciari'1, Tracey Varker², Grant J. Devilly ${ }^{3 *}$ \\ Running Head: Neural Impact of Trauma
}

Keywords: Neural Connectivity, EEG Coherence, PTSD, Developmental, Trauma

Word count (excluding references, abstract and tables \& Figures): 3,700

1. Brain Sciences Institute, Swinburne University.

2. Australian Centre for Post-traumatic Mental Health, University of Melbourne

3. School of Psychology \& Griffith Institute for Health and Medical Research, Griffith University

\footnotetext{
*Address for Correspondence

Prof. Grant J. Devilly, PhD.

School of Psychology \& Griffith Institute for Health and Medical Research, Griffith University - MtGravatt Campus, Messines Ridge Road, Mt Gravatt, Qld 4122. Australia.

Email: g.devilly@griffith.edu.au
}

Acknowledgements: We would like to acknowledge the input of Christie Turton for her assistance in acquiring data. 


\begin{abstract}
Objective: Neural connectivity differences between adults reporting childhood, adulthood or no history of trauma were examined. Methods: A total of 39 participants completed the Posttraumatic Stress Diagnostic Scale (PDS; Foa, 1995), a Word Memory Task (WMT; McNally, Metzger, Lasko, Clancy, \& Pitman, 1998) and EEG analysis. Intelligence was not assessed during the study. Results: As predicted, those with childhood trauma had significantly higher EEG coherence than those with either adulthood trauma or no past trauma. Conclusions: Significant differences were observed over frontal, central, temporal and parietal areas. Evidence was found suggesting that childhood psychological trauma may have a lasting impact on neuronal connectivity. Significance: This is the first study to demonstrate the suspected long term effect of trauma over central, temporal and parietal areas. Long term neural correlates of childhood and adult trauma appear to suggest information processing differences - differences that may, eventually, lead to better interventions following trauma.
\end{abstract}


The relationship between childhood traumatic experiences and increased prevalence of mental illness throughout the lifespan is well documented (eg., Watts-English et. al., 2006). Neuropsychological research has provided evidence that changes in catecholamine levels following a traumatic event can impede brain region development, which in turn can compromise later cognitive functioning and leave a person susceptible to mental illness. This forms the basis of the theory of developmental traumatology (De Bellis, 2001). If the brain undergoes a prolonged state of hyperarousal during the maturation of limbic system areas, it can develop inappropriate and maladaptive neural networks that may put traumatized children at increased risk of subsequent mental illness (Felitti et. al., 1998; Mulvihill, 2005).

The processes of synaptic pruning, myelination and neurogenesis are most active until around age 10 when the brain reaches the weight of an adult brain (Giedd et al., 1999; Paus et. al., 2001; Pfefferbaum et. al., 1994; Sowell et.al., 2002). As the body reaches adolescence these processes begin to slow down and neural networks become somewhat more fixed, although, in some areas, these processes continue into the third decade of life (Teicher et. al., 1997).

An unbalanced catecholaminergic system can influence the brain's metabolism, slowing or accelerating rates of pruning and myelination (Weber, \& Reynolds, 2004). Several studies have found abnormal catecholamine levels in those who have been witness to trauma (e.g., Yehuda, 1997, 2000). When a child, of any age, is subjected to a traumatic stressor, it is widely believed that inappropriate levels of catecholamines can be responsible for delayed and abnormal neural development (De Bellis, 2001; Weber \& Reynolds, 2004). As a full review of this area is outside the scope of this paper, we guide the reader to the work of De Bellis (2001) and Yehuda (2000). 
Several studies utilizing Magnetic Resonance Imaging (MRI) in maltreated children have identified structures that appear to be adversely affected by traumatic stress. De Bellis and colleagues (De Bellis \& Keshavan, 2003; De Bellis \& Kuchibhatla, 2006; De Bellis et. al., 2002) have demonstrated in children with maltreatment related PTSD, significantly smaller volumes in the cerebral and prefrontal cortex, temporal lobes, corpus callosum and cerebellum. Specifically, in De Bellis’ 2006 study, cerebellar volumes were positively related to age of onset of trauma; the earlier the age of trauma, the smaller the cerebellar volume of the child. These findings strongly suggest that trauma is related to arrested neural development and growth of brain structures, which in turn could explain the high prevalence of mental illness (Felitti et. al., 1998; Mulvihill, 2005) and cognitive deficits (Navalta et.al., 2006) reported in this group.

In an earlier study, Ito et al. (1998) utilized EEG coherence to examine whether the connectivity of neural structures and networks are in fact abnormal in traumatized children. Severely sexually/physically-abused children were compared to non-abused controls. Fifteen abused children aged 6 to 15 years and 15 non-abused controls each underwent EEG assessment. An eight-minute period of EEG was recorded while participants rested with their eyes closed. From these eight minutes, 40 seconds of EEG was selected for further analysis. Intrahemispheric coherence was computed only for the alpha band and the coherence of all combinations of lateral electrodes in each hemisphere of the brain was calculated.

Left hemisphere coherence was found to be significantly greater in the abused group than the controls, and specific asymmetries were identified in areas over the central, temporal and parietal regions of the brain. It was suggested that these asymmetries were due to reduced left hemisphere cortical differentiation of signals, due to ample myelination of neurons and reduced complexity of synaptic specialisation. 
It is also possible that the EEG coherence may be mediated by a common sub-cortical structure driving two or more cortical areas (French \& Beaumont, 1984; Petsche, 1997). If this is the case, EEG coherence measures may be useful in detecting deeper neural changes associated with the experiencing of traumatic stress, such as those that have already been demonstrated in MRI studies (De Bellis \& Keshavan, 2003; De Bellis \& Kuchibhatla, 2006; De Bellis et. al., 2002).

Overall, the EEG findings of Ito and colleagues (1998) suggest that early trauma has a significant adverse impact on the central, temporal and parietal regions of the child or adolescent brain. The differences observed between groups in neuronal connectivity may be instructive in explaining the tendency for traumatized children to suffer increased prevalence of mental illness and cognitive deficits. It would seem that the arrangement of neural networks in traumatized children are maladaptive or impeded, and are not as capable of providing healthy functioning as the networks observed in healthy controls. It is unknown whether these neural network deficits remain present into adulthood.

The current study aimed to examine the occurrence of past trauma in an adult sample of the population and investigate whether differences in EEG coherence exist between those with childhood, adulthood, and those with no past trauma. The theory of developmental traumatology suggests that early impedance of brain development can have lasting cognitive effects into adulthood. If these effects are in fact lasting, we could then assume that adults reporting childhood traumas must have a somewhat altered neural layout. We, therefore, assumed that the coherence patterns of adults reporting childhood traumas would be somewhat similar to those observed by Ito et al., in their sample of traumatized children. That is, if neural development is altered in traumatized children then differences will be observable in traumatized adults. Based on Ito et. al.'s (1998) findings, it was hypothesized that adults reporting childhood traumas would 
have higher coherence in electrode pairs over central, temporal and parietal areas, than adults reporting either adulthood trauma, or no past trauma history.

Method

\section{Participants}

A total of 39 participants took part in the study; 20 were females (Age $M=21.55$, $S D=2.98$ ) and 19 males (Age $M=20.58, S D=2.98$ ), All were recruited from a university community.

For the purposes of the EEG analysis, only participants with no history of head trauma, no epilepsy, and no family history of epilepsy were included in the study. No participant reported psychiatric illness. There was also a seven-day drug free period before testing. Participants taking oral contraceptives or occasional paracetamol were still included in the study. Participants were not allowed to consume alcohol for 24 hours beforehand or caffeine for 3 hours beforehand. There were no blood tests carried out to confirm the absence of drugs, we simply asked participants upon arrival for testing whether they had conformed to the 7 day wash out period and all reported that they had.

Participants were selected to take part in this research based upon them scoring as either high $(>15)$ or low $(<10)$ on the Dissociative Experiences Scale (DES; Bernstein-Carlson, 1986). This inclusion criteria aimed to allow for a replication of other research investigating memory deficits in high dissociators (DePrince \& Freyd, 2004) which has been published elsewhere (Devilly et. al., 2007; Devilly \& Ciorciari, 2007). This other research had a group cut off for the high DES group of 20 and above and hence excluded 2 participants (those scoring between 15 and 19). For the purpose of the current study these 2 participants were included in analyses as 
DES score was subsequently found to have no impact on alpha or beta bandwidth EEG analyses (see supplementary results) and was not the focus of the current research.

\section{Materials}

The Post-traumatic Stress Diagnostic Scale (PDS: Foa, 1995) was used to assess the occurrence of past traumatic experiences. The PDS examines the occurrence of trauma, and the presence and severity of PTSD symptoms and any subsequent impairment. The psychometric properties of the PDS are well established. Cronbach's alpha for the total PDS score has been reported as .91, with high test-retest reliability of .74 when assessed one month later (Foa et. al., 1993).

The Word Memory Task (WMT; McNally et. al., 1998) was used to induce a very actively focused cognitive state during the EEG recordings and it was also thought to be a task which would activate the specific brain regions under investigation (Haarmann \& Cameron, 2005; Haarmann et. al., 2002). The WMT assesses participants' recall and recognition of words under selective attention, divided attention with motor task (moderate difficulty), and divided attention with verbal task (very difficult) conditions. Three blocks of words were utilised each with its own set condition. Each block comprised of six trauma related words (e.g., rape), six positive words (e.g., happiness) and six neutral words (e.g., desk), to form a total of 18 words in each block. Words were presented for 2 seconds each with the command to either remember (“RRRR”) or forget ("FFFF”) appearing for 2 seconds after each presented word. Block one was a selective attention task requiring participants to only obey commands to either remember or forget each word. Block two was the divided attention with motor response task that required marking a piece of paper if word colour changed, while obeying commands to either remember or forget. Block three was the divided attention verbal task requiring that participants count backwards from 200, by three's, out loud while also obeying the commands to either remember 
or forget each word. Participants viewed each block only once as this was thought to reflect the 'real world' more accurately than the three presentations of each block that was previously used by DePrince and Freyd (2001). Block order was randomised amongst participants. Verbal instructions were given before each block by the researcher who remained present in the room to ensure the task was completed properly. After viewing all three blocks participants were asked to freely recall as many words as possible, regardless of whether they were told to remember or forget each word. A recognition task required participants to tick off any words they believed they had seen, again irrespective of whether they were told to remember or forget each word, from a list of words that included 54 distractor words that did not appear during any of the blocks.

EEG was recorded for 10 seconds immediately after the last word was presented from the WMT and before the recall command. Participants were fitted with a 32 channel EEG cap (International 10/20 system placement) which was connected to a Neuroscan EEG amplifier \& recording system. Mastoid and nose reference electrodes and forehead ground were used. An electro-oculargram (EOG) was also recorded for artifact rejection during offline analyses. The total gain (amplification) was 100k with bandwidth filters set at $.15 \mathrm{~Hz}$ and $50 \mathrm{~Hz}$, with the intention of eliminating muscle artifact. The data acquisition rate was set at $1000 \mathrm{~Hz}$., with all electrode impedances below $5 \mathrm{k} \Omega$. Neuroscan acquisition software (SCAN version 4.2) was used to record, digitize and process EEG data. When the participants finished the WMT, their typical reaction was to blink a few times and move their face, providing large muscle activity artifact in the EEG recordings. We, therefore, visually screened the data and selected only the first five artifact free seconds of data following this muscle interference. It was this 5 second epoch that underwent averaging, spectral analysis and subsequent coherence computation. This single five second epoch of time used for the analyses is longer than those typically used in other studies 
(e.g., Jiang \& Zheng, 2006) and allows for greater confidence in the results. During off-line analyses the Neuroscan EDIT analysis software was used to remove artifacts and calculate the alpha $(8-14 \mathrm{~Hz})$ and beta $(14-30 \mathrm{~Hz})$ coherence. It is believed this initial five seconds after viewing word stimuli would be a period of heightened cognitive activity, while words were held in memory. For off-line analyses, a 16 electrode pair interhemispheric and intrahemispheric montage was applied, with pairs focused over frontal, central, temporal and parietal areas. Electrode pairs selected were the following: F8-F7, FC3-F7, FC4-F8, FZ-FT7, FT8-FZ, FT8FT7, FCZ-FZ, FT7-FC3, FC4-FT8, C3-T7, C4-T8, CPZ-CZ, TP8-T7, T8-TP7, CP3-T7, CP4-T8. This montage is displayed in Figure 1 (A). Effect size analyses were conducted using ClinTools Software Version 4 (Devilly, 2007), and all other analyses were carried out using SPSS version 14.0.0 (SPSS Inc., 2005) and Statistica version 6.1 (Statsoft, 2003).

\section{INSERT FIGURE 1 HERE}

\section{Procedure}

The PDS was administered prior to EEG setup. Childhood trauma was classified as trauma occurring under the age of 16 years old. This cut-off was chosen for consistency with Teicher et al (1997) and also due to the fact that the age of consent in Victoria, Australia, is 16 years old. Following EEG preparation, participants were verbally informed of the requirements of the WMT before randomly receiving all three blocks of trauma, positive and neutral words. Prior to the presentation of the final block of words, participants were instructed that when the computer screen went blank at the end of the word presentation they would be required to sit still looking at the black screen for 10 seconds, while holding the presented words in memory and focusing their attention on them. EEG recordings were taken throughout this 10 -second period. Participants then completed the free recall and recognition tasks.

Results 
As explained above, data from the PDS was used to divide participants into three groups: those reporting the occurrence of childhood trauma (CT), those reporting adulthood trauma (AT), and those reporting no past trauma (NT). A single person reporting both CT and AT was excluded from the analyses. Group numbers were relatively equal (CT=13; AT=13; NT=12) and gender distribution was not significantly different between groups (CT: female $=6$, male $=7$; AT: female $=10$, male $=3$; NT: female $=4$, male $=8 ; \chi^{2}(\mathrm{df}=2)=5.09, p<.08$; Fisher's Exact Test $p<.09$ ). Mean age at the time of the traumatic event for the CT group was $M=11.77(S D=3.03)$ and for the AT group was $M=19.38(S D=2.69)$ representing a significant difference $(F(1,24)=45.83$, $p<.001$ ). Mean amount of time elapsed since trauma (measured in months) for the child trauma group was $M=98.23(S D=36.29)$, and for the adult trauma group was $M=30.88(S D=26.67)$. This was a significant difference $(F(1,24)=29.07, p<.001)$ with a very large effect size (Hedges' $\hat{g}=2.1$, 95\%CI: 1.1-3.0). Current participant ages were not significantly different between groups (CT: $M=20, S D=1.53$; AT: $M=21.92, S D=2.9$; NT: $M=21.42, S D=3.75)$. The most common type of trauma reported for both CT and AT groups was the experiencing of the death of someone close (CT=6, AT=4), with other varied forms of trauma reported including rape, witnessing a life threatening accident and experiencing a life threatening illness. This breakdown is presented in Table 1. In addition, a t-test revealed that there were no significant differences between CT and AT trauma groups in either level of distress experienced at time of the index trauma $(t(24)=1.01$, $n s)$, or level of distress currently experienced due to index trauma $(t(24)=-0.81, n s)$.

\section{INSERT TABLE 1 ABOUT HERE}

Performance on the WMT was compared across trauma groups. The total number of trauma, positive and neutral words that were correctly recalled and recognized for both 'remember' and 'forget' conditions was similar across childhood trauma, adulthood trauma and no past trauma groups. This indicated that, as planned, the WMT provided a similarly focused 
mental state across groups. Comprehensive analyses of the WMT task results, delimited by dissociative propensity and history of trauma, have been published elsewhere (Devilly et al., 2007; Devilly \& Ciorciari, 2007).

A 3 (Childhood trauma, Adulthood trauma, no past trauma) by 16 (electrode pairs) ANOVA was conducted for the alpha and beta bandwidths. These specific electrode pairs and frequency bandwidths were examined due to the already introduced hypotheses based upon Teicher et al. (1997), Ito et al. (1998) and Hooper et al. (2002). Significant differences were found between CT and AT groups and CT and NT groups (Table 2). Hedges $\hat{g}$ effect sizes are indicated with the lowest value of 1.01, indicating overall strong effects, although the $95 \%$ confidence intervals (also shown) are quite large. A correction was made due to the high number of analyses required. Balancing out Type I and Type II error, the significance level was set at $p<.01$, so that only one in 100 analyses would appear significant by chance. Given that 7 significant differences were found from a total of 32 analyses, it would be reasonable to assume that the differences observed were not found by chance alone.

\section{INSERT TABLE 2 ABOUT HERE}

As indicated in Table 2, those with a history of childhood trauma had significantly higher alpha coherence in electrode pairs over right sided frontal and temporal areas, and higher beta coherence over right temporal-parietal areas, than those with adulthood traumas. Those with childhood traumas also had significantly higher alpha coherence over left temporal-parietal areas and higher beta coherence over the right temporal region, than those with no trauma history. Pictorial representations of significant electrode pairs are presented in Figure 1 (B \& C). Although outside of the corrected significance alpha level, it may also prove worthwhile in future research to note that, in comparing the difference between the Childhood Trauma group to the No 
Trauma group, there was one right hemisphere electrode pair (FT8-FZ) of the alpha range that approached significance $(p=.013)$.

Supplementary Results

Coherence between the significant electrode pairs was correlated with length of time since trauma (measured in months). A significant positive correlation was found for four of the six significant electrode pairs (Alpha FC4-FT8, rho=.617, $p=.001$; Alpha FC4-F8, rho=.494, $p=.01$; Alpha FT8-FZ, rho=.673, $p<.01$; Beta C4-T8, rho=.442, $p<.05)$. As the current age of participants was similar with a small standard deviation $(M=21.08, S D=2.86)$, this correlation indicated that, in general, the higher the total number of months since trauma (i.e., the younger the age of trauma), the higher the coherence between significant electrode pairs. In order to ascertain whether coherence differences between trauma groups were more likely due to the time period during which the trauma occurred (childhood or adulthood) rather than the time passed since trauma, Spearman's rank correlations were performed between 'months since trauma' and coherence values within each trauma group. No significant correlations within trauma group were observed, indicating that differences in coherence between groups were most likely due to the time period of the trauma rather than time since trauma.

An analysis of total DES score was carried out to determine whether dissociation had any influence on EEG coherence measures. A 2 (DES: high and low groups) by 6 (significant electrode pairs differentiating adult $\mathrm{v}$ childhood trauma) ANOVA revealed no significant differences in EEG coherence between groups. In addition, no significant correlations were observed between total DES score and EEG coherence, indicating that the DES grouping had no influence on the results - an outcome which raises further questions for researchers who hypothesize DES score as a mediator of memory style and childhood trauma experiences.

Discussion 
Support was found for the hypothesis that adults reporting childhood trauma would show higher coherence in central, temporal and parietal regions than those with either adulthood trauma or no trauma history. Specifically, those reporting childhood trauma had significantly higher coherence of the alpha band over right hemisphere frontal, central and temporal areas, and of the beta band in the right central, temporal parietal areas, than those reporting adulthood traumas. Also, those reporting childhood traumas had significantly higher alpha coherence over the left temporal, central and parietal regions and higher beta coherence over right hemisphere central temporal areas, than those with no history of trauma.

Correlations revealed that coherence had a tendency to be higher in those with earlier traumas generally, which is consistent with past findings (Ito et al., 1998). Further within group correlations suggested that differences in coherence between groups were more likely due to the time period that the trauma occurred, rather than the time passed since the trauma. This supports De Bellis’ (2006) results suggesting that earlier traumas can have a more severe impact on neural development than traumas occurring later on in childhood.

The current results support Ito et al (1998) and suggest that an altered neural development during childhood may leave a lasting imprint on neural networks that can be measured even in adulthood. It is possible that these differences in neural connectivity in part contribute to an increased tendency for those reporting childhood trauma to experience mental illness. This is in keeping with the theory of developmental traumatology (De Bellis, 2001). The specific impact these neural differences have on adult cognitive functioning is yet to be identified. Interestingly, the sample of adults we tested who reported childhood trauma did not differ in Word Memory Task performance in comparison to Adulthood Trauma or those with no trauma history. It may be that any cognitive deficits present in the Childhood Trauma group were simply not specific to memory or word identification performance that were examined by this task. It may also be that 
while coherence differences between groups were significantly different, the impact of these differences on cognitive functioning were so subtle as to not reach significance at all. It is also possible that, as all our subjects had received advanced schooling to University level, this may have acted as a buffer to cognitive deficits, or we had participants who had learned compensatory strategies. Comprehensive longitudinal studies are required to make any further assumptions about this potential cause-consequence relationship.

The EEG was recorded after the word presentation, before participants were asked to recall as many words as possible. We assume that this would have provoked the activation of language and emotion centers, given the emotional content of the presented words (Hwang et. al., 2005) Differences in coherence between groups occurred in the temporal and frontal areas known to be responsible for these cognitive functions.

The observed increases in right frontal coherence may reflect negative emotion processing differences between those with different trauma histories, which could have been provoked by the viewing of trauma related words in the WMT. The increased EEG coherence correlations observed in the frontal regions may reflect different strategies used to maintain words in working memory, or possibly a different level of analysis of word meaning. In a study examining coherence changes during active maintenance of a sentence in working memory, similar synchronization of the frontal cortex has been observed (Haarmann \& Cameron, 2005).

Altered catecholamine functioning following trauma can influence neural development and could be responsible not only for impeding development but also for increasing the risk of mental illness throughout the lifespan (De Bellis, 2001). Research examining reduced brain volumes in those with a childhood trauma history has provided tentative evidence of the severe effects that stress can have on the growth of the brain. The current results suggest that there is different neural connectivity in those with childhood traumas. It could be assumed that this is due 
to altered catecholamine levels and hence inappropriate brain metabolism during important, formative years. A longitudinal study examining catecholamine levels, structural volume, and occurrence of mental illness is required to confirm this assertion.

Those with higher than average intelligence have been shown to have significantly lower coherence of the alpha band than those with average intelligence (Jausovec \& Jausovec, 2005) and it has been suggested that this may reflect faster, more efficient, processing of information. Alpha activity has been associated with performance of cognitive tasks (Sauseng et. al., 2005) such as that of the WMT. If those with lower intelligence have less differentiation of signals, it is possible that information is not being adequately assessed, considered, compared, or scrutinized in as much detail as those with high intelligence. This processing may also be less efficient.

Further, research has suggested that intelligence may act as a protective factor against PTSD. Research into war veterans indicated that those with a lower IQ displayed a significantly higher severity of PTSD symptoms than those with a higher IQ (McNally \& Shin, 1995). Similar findings have been observed in several instances (Hunt \& Evans, 2004; Macklin et al., 1998; Vasterling et al., 2002).

Based on these findings, it is possible that the EEG coherence estimates observed in the current study may reflect intelligence levels. Those with childhood traumas exhibited increased coherence of the alpha band, much like what we might expect in a sample of people scoring lower on intelligence. Perhaps childhood trauma restricted normal neurodevelopment, as suggested by Ito et al (1998), and resulted in delayed or impaired cognitive development. It is also possible that those low on intelligence were simply more likely to report an event as being traumatic because it affected them more than the same event may have affected those higher on intelligence. Only longitudinal studies will allow us to escape the cause-consequence conundrum. 
The current study was the first of its kind and participant numbers were limited. Also, participants were relatively young with most aged in their early twenties. However, age homogeneity was needed for this first study to counteract aging explanations of any possible results.

There is evidence that neural development in some brain regions continues into the third decade of life (Teicher et. al., 1997) and the authors acknowledge that the sample used is not representative of all adults in general. As expected, the reported adulthood traumas had occurred more recently than the childhood traumas, making it possible that any effects of adulthood trauma had not yet had time to manifest in this group. However, correlational data suggested that it was more likely that the time of trauma, rather than the time elapsed since trauma, explained the differences between groups on coherence measures. It is also possible that these adulthood traumas simply occurred late enough during neural development, for the effects of trauma on neural networks to be far less (or even for there to be no effect on neural networks at all) than if they had occurred at a younger, more sensitive stage of development. Interestingly, the adult trauma and no past trauma groups were not significantly different in coherence measures, indicating that underlying neural networks must be similar. Teicher et al (1997) suggest that by adolescence neural networks are somewhat more fixed.

It is outside the scope of this research to form any firm conclusions as to which specific age groups are most vulnerable to the neurological consequences of traumatic experiences, or what specific processes are responsible for any such differences between groups. This research has, however, pointed out that a difference in EEG coherence can be observed between adults reporting adulthood traumas and adults reporting childhood traumas. We see that an extension of this study, with a larger sample displaying a greater age range, would now be appropriate as a next step. A larger sample would also allow for comparisons of the effects of differing types of 
trauma. We would also suggest that future studies assess intelligence as measured by formalized IQ strategies and control for this variable where necessary.

There are many factors, due to the influences of both nature and nurture that may affect the development of neural areas. It has been suggested that neural abnormalities reported in traumatized participants could be genetically inherited, and these could put people at higher risk of trauma exposure (Teicher et. al., 1997). Longitudinal studies will be required to form any definite conclusions in this debate.

Genders within groups were not balanced, and the authors acknowledge that this may have impacted on EEG measures. Analyses were only correlational in nature and, therefore, can only point out relationships within the data. It is possible that coherence differences were a result of two areas receiving the same input from other neural structures (Nunez et al., 1997). However, as there were significant differences between groups, we can at least be sure that some differences in processing do occur between different trauma groups during a working memory task, the specific causes of which still need clarification

In conclusion, EEG coherence measures indicated significantly different patterns between those with childhood, adulthood or no past trauma. This adds to the growing body of evidence that trauma can have a lasting impact on neural connectivity - a result which future treatment studies may be able to exploit. 


\section{References}

Bernstein-Carlson E B, Putnam FW. Development, reliability and validity of a dissociation scale. J Nerv Ment Dis. 1986; 174: 727-735.

De Bellis MD. Developmental traumatology: The psychobiological development of maltreated children and its implications for research, treatment, and policy. Dev Psychopathol. 2001; 13, 539-564.

De Bellis MD, Keshavan MS. Sex differences in brain maturation in maltreatment-related pediatric posttraumatic stress disorder. Neurosci Biobehav Rev. 2003; 27, 103-117.

De Bellis MD, Kuchibhatla M. Cerebellar volumes in pediatric maltreatment-related posttraumatic stress disorder. Biol Psychiatry. 2006; 60, 697-703.

De Bellis MD, Keshavan M, Shifflett H, Iyengar S, Beers SR, Hall J, et. al. Brain structures in pediatric maltreatment-related PTSD: A sociodemographically matched study. Biol Psychiatry. 2002; 52, 1066-1078.

DePrince AP, Freyd JJ. Forgetting trauma stimuli. Psychological Science. 2004; 15, 488-492.

Devilly, G.J. (2007). ClinTools Software for Windows: Version 4.1 (computer programme). www.clintools.com. Melbourne, Australia.

Devilly GJ, Ciorciari J. Conclusions in science when theory and data collide. Psychological Science. 2007; 18, 220-221.

Devilly GJ, Ciorciari J, Piesse A, Sherwell S, Zammit S, Cook F, Turton C. Dissociative tendencies and memory performance on directed forgetting tasks. Psychological Science. 2007; 18, 212-217.

Felitti VJ, Anda RF, Nordenberg D, Williamson DF, Spitz AM, Edwards V, et al. Relationship of childhood abuse and household dysfunction to many of the leading causes of death in 
adults: The adverse childhood experiences (ACE) study. Am J Prev Med. 1998; 14, 245258.

Foa EB. Posttraumatic Stress Diagnostic Scale (PDS) Manual. Minneapolis, MN: National Computer Systems, 1995.

Foa EB, Riggs DS, Dancu CV, Rothbaum BO. Reliability and validity of a brief instrument for assessing post-traumatic stress disorder. J Trauma Stress. 1993; 6, 459-473.

French CC, Beaumont JG. A critical review of EEG coherence studies of hemisphere function. Int J Psychophysiol 1984; 1: 241-254.

Giedd JN, Blumenthal J, Jeffries NO, Castellanos FX, Liu H, Zijdenbos A, et al. Brain development during childhood and adolescence: A longitudinal MRI study. Nature Neuroscience. 1999; 2, 861-863.

Haarmann HJ, Cameron KA. Active maintenance of sentence meaning in working memory: Evidence from EEG coherences. Int J Psychophysiol. 2005; 57, 115-128.

Haarmann HJ, Cameron KA, Ruchkin DS. Neural synchronization mediates on-line sentence processing: EEG coherence evidence from filler-gap constructions. Psychophysiology. 2002; 39, 820-825.

Hopper A, Ciorciari J, Johnson G, Spensley J, Sergejew A, Stough C. EEG coherence and dissociative identity disorder. Journal of Dissociation and Trauma. 2002; 3:1.

Hunt N, Evans D. Predicting traumatic stress using emotional intelligence. Behav Res Ther. 2004; 42, 791-798.

Hwang G, Jacobs J, Geller A, Danker J, Sekuler R, Kahana MJ. EEG correlates of verbal and nonverbal working memory. Behav Brain Funct. 2005; 1. 
Ito $\mathrm{Y}$, Teicher MH, Glod CA, Ackerman E. Preliminary evidence for aberrant cortical development in abused children: A quantitative EEG study. J Neuropsychiatry Clin Neurosci. 1998; 10, 298-307.

Jiang ZY, Zheng LL. Inter-and intra-hemispheric EEG coherence in patients with mild cognitive impairment at rest and during working memory task. Journal of Zhejiang University Science B. 2006; 7, 357-364.

Jausovec N, Jausovec K. Differences in induced gamma and upper alpha oscillations in the human brain related to verbal/performance and emotional intelligence. Int $\mathrm{J}$ Psychophysiol. 2005; 56, 223-235.

Joseph R. Environmental influences on neural plasticity, the limbic system, emotional development and attachment: A review. Child Psychiatry Hum Dev. 1998; 29, 189-208.

Macklin ML, Metzger LJ, Litz BT, McNally RJ, Lasko NB, Orr SP, et al. Lower precombat intelligence is a risk factor for posttraumatic stress disorder. J Consult Clin Psychol. 1998; 66, 323-326.

McNally R J, Metzger LJ, Lasko NB, Clancy SA, Pitman RK. Directed forgetting of trauma cues in adult survivors of childhood sexual abuse with and without posttraumatic stress disorder. J Abnorm Psychol. 1998; 107, 596-601.

McNally RJ, Shin LM. Association of intelligence with severity of posttraumatic stress disorder symptoms in Vietnam combat veterans. Am J Psychiatry. 1995; 152, 936-938.

Mulvihill D. The health impact of childhood trauma: An interdisciplinary review, 1997-2003. Issues in Comprehensive Pediatric Nursing. 2005; 28, 115-136.

Navalta CP, Polcari A, Webster DM, Boghossian A, Teicher MH. Effects of childhood sexual abuse on neuropsychological and cognitive function in college women. J Neuropsychiatry Clin Neurosci. 2006; 18, 45-53. 
Nunez PL, Srinivasan R, Westdorp AF, Wijesinghe RS, Tucker DM, Silberstein RB, et al. EEG coherency I: Statistics, reference electrode, volume conduction, Laplacians, cortical imaging, and interpretation at multiple scales. Electroencephalogr Clin Neurophysiol.1997; 103, 499-515.

Paus T, Collins DL, Evans AC, Leonard G, Pike B, Zijdenbos A. Maturation of white matter in the human brain: A review of Magnetic resonance studies. Brain Res Bull. 2001; 54, 255266.

Petsche H. EEG coherence and mental activity. In Angeleri F, Butler S, Giaquinto S, Majkowski J, editors. Analysis of the electrical activity of the brain. New York: John Wiley \& Sons, 1997: 141-166.

Pfefferbaum A, Mathalon DH, Sullivan EV, Rawles JM, Zipursky RB, Lim KO. A quantitative magnetic resonance imaging study of changes in brain morphology from infancy to adulthood. Arch Neurol. 1994; 51, 874-887.

Sarnthein J, Morel A, von Stein A, Jeanmonod D. Thalamocortical theta coherence in neurologic patients at rest and during a working memory task. Int J Psychophysiol. 2005; 57, 87-96.

Sauseng P, Klimesch W, Schabus M, Doppelmayr M. Fronto-parietal EEG coherence in theta and upper alpha reflect central executive functions of working memory. Int $\mathrm{J}$ Psychophysiol. 2005; 57, 97-103.

Sowell ER, Trauner DA, Gamst A, Jernigan TL. Development of cortical and sub cortical brain strustures in childhood and adolescence: A structural MRI study. Dev Med Child Neurol. 2002; 44, 4-16.

SPSS for Windows, Rel. 14.0.0. 2005. Chicago: SPSS Inc.

StatSoft, Inc. (2003). STATISTICA (data analysis software system), version 6. www.statsoft.com, accessed $8^{\text {th }}$ November 2007. 
Teicher MH, Ito Y, Glod CA, Anderson SL, Dumont N, Ackerman E. Preliminary evidence for abnormal cortical development in physically and sexually abused children using EEG coherence and MRI. New York: New York Academy of Sciences. (1997).

Vasterling JJ, Duke LM, Brailey K, Constans JI, Allain AN, Sutker PB. Attention, learning, and memory performances and intellectual resources in vietnam veterans: PTSD and no disorder comparisons. Neuropsychol Rev. 2002; 16, 5-14.

Watts-English T, Fortson BL, Gibler N, Hooper SR, De Bellis MD. The psychobiology of maltreatment in childhood. Journal of Social Issues. 2006; 62, 717-736.

Weber DA, Reynolds CR. Clinical perspectives on neurobiological effects of psychological trauma. Neuropsychol Rev. 2004; 14, 115-129.

Yehuda R. Sensitization of the hypothalamic-pituitary-adrenal axis in posttraumatic stress disorder (Vol. 821). New York: New York Academy of Sciences, 1997.

Yehuda R. Cortisol alterations in PTSD, International Handbook of Human Response to Trauma. New York: Plenum, 2000, 265-283. 
Table 1. Index Trauma Types Experienced by Participants and Mean Severity Ratings as SelfJudged "at the time" and "currently".

\begin{tabular}{|c|c|c|}
\hline Measure & CT $(\mathrm{N}=13)$ & AT $(\mathrm{N}=13)$ \\
\hline \multicolumn{3}{|l|}{ Index Trauma } \\
\hline Parent Dying & 1 & \\
\hline Someone close dying not unexpectedly & 2 & 1 \\
\hline Very serious mental/physical illness & 1 & 2 \\
\hline Emotional abuse/neglect & 1 & \\
\hline Parents separating/divorcing & 2 & \\
\hline Someone close unexpectedly dying & 3 & 3 \\
\hline Hijacking & 1 & \\
\hline Witnessing Trauma & 1 & \\
\hline Rape & & 1 \\
\hline Physically threatened in a confined space & & 1 \\
\hline Separation or divorce & 1 & 1 \\
\hline Mentally ill/suicidal sister & & 1 \\
\hline Being in or witnessed combat & & 1 \\
\hline Witnessed a life threatening accident & & 1 \\
\hline Sister had a stroke & & 1 \\
\hline Mean (sd) distress at time of index trauma & $4.15(.80)$ & 3.77 (1.09) \\
\hline Mean (sd) current distress from index trauma & $1.67(.65)$ & $1.92(.90)$ \\
\hline
\end{tabular}

Note: At = Adult Trauma; $\mathrm{CT}=$ Childhood Trauma 
Table 2. Mean Coherence Values for the Significant Electrode Pairs (Tukey HSD) Between CT, AT and NT Groups, with Standard Deviations, Effect Sizes and 95\% Confidence Intervals.

\begin{tabular}{|c|c|c|c|c|c|c|c|c|}
\hline Comparison & Bandwidth & $\begin{array}{l}\text { Electrode } \\
\text { Pairs }\end{array}$ & $\begin{array}{c}\text { AT } \\
\mathrm{N}=13\end{array}$ & $\begin{array}{c}\mathrm{CT} \\
\mathrm{N}=13\end{array}$ & $\begin{array}{c}\mathrm{NT} \\
\mathrm{N}=12\end{array}$ & Hedges $\hat{g}$ & $\begin{array}{l}\text { 95\% } \\
\text { Lower }\end{array}$ & $\begin{array}{l}\text { 95\% } \\
\text { Upper }\end{array}$ \\
\hline \multirow[t]{5}{*}{ CT vs. AT } & Alpha & *FC4-FT8 & $.67(0.18)$ & $.85(0.12)$ & - & 1.14 & .31 & 1.97 \\
\hline & & *FC4-F8 & $.65(0.14)$ & $.79(0.13)$ & - & 1.01 & .19 & 1.83 \\
\hline & & *FT8-FZ & $.63(0.13)$ & $.79(0.11)$ & - & 1.26 & .42 & 2.10 \\
\hline & Beta & *CP4-T8 & $.47(0.10)$ & $.61(0.13)$ & - & 1.07 & .25 & 1.90 \\
\hline & & ${ }^{*} \mathrm{C} 4-\mathrm{T} 8$ & $.50(0.10)$ & $.64(0.13)$ & - & 1.14 & .31 & 1.97 \\
\hline \multirow[t]{2}{*}{ CT vs. NT } & Alpha & *CP3-T7 & - & $.71(0.16)$ & $.50(0.16)$ & 1.19 & .34 & 2.04 \\
\hline & Beta & *C4-T8 & - & $.64(0.13)$ & $.49(0.13)$ & 1.09 & .25 & 1.93 \\
\hline
\end{tabular}

Note: *Significant at $p<.01$, CT= Childhood Trauma Group, AT= Adulthood Trauma Group, NT= No Past Trauma Group. 
Figure 1. EEG coherence montage (A) and resulting pairs found to be significantly different ( $p<.01$ ) between trauma groups (B \& C)

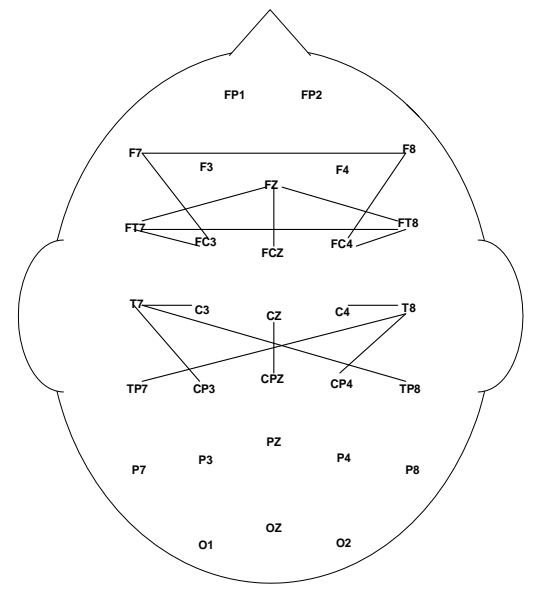

A. EEG coherence montage

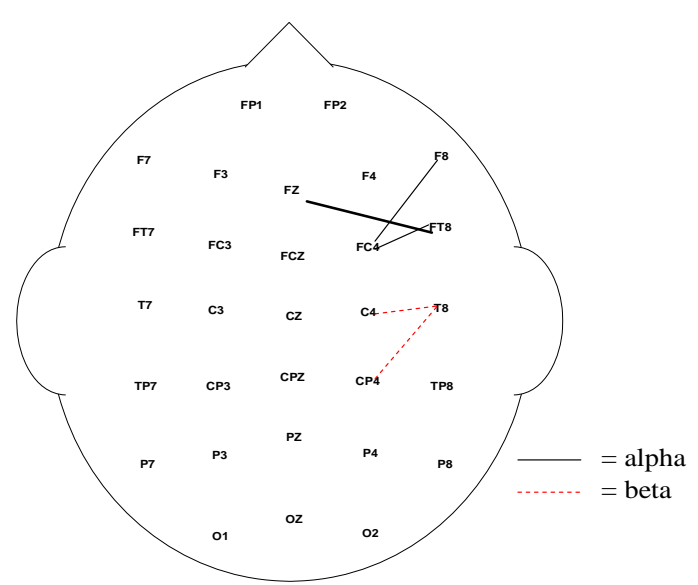

B. CT group higher coherence than AT group $(p<.01)$

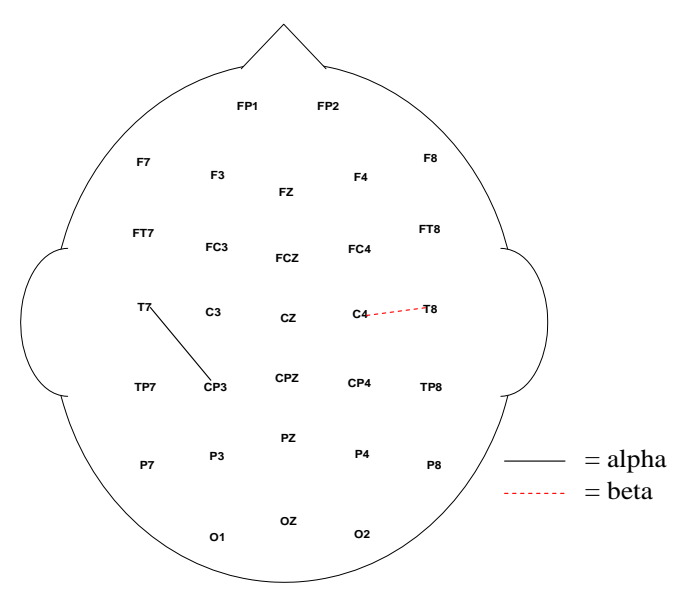

C. CT group higher coherence than NT group $(p<.01)$

*Note: CT= Childhood Trauma, AT= Adulthood Trauma, NT= No Past Trauma 